\title{
Constituintes químicos e avaliação das atividades antibacteriana e antiedematogênica de Aloysia gratissima (Gillies \& Hook.) Tronc. e Aloysia virgata (Ruiz \& Pav.) Pers., Verbenaceae
}

\author{
Fábio Vandresen, ${ }^{1}$ Elisângela Schmitt, ${ }^{1}$ Lucília Kato, ${ }^{2}$ Cecília Maria Alves de Oliveira, ${ }^{2}$ \\ Ciomar Aparecida Bersani Amado, ${ }^{3}$ Cleuza Conceição da Silva ${ }^{*}, 1$
}

\author{
${ }^{1}$ Departamento de Química, Universidade Estadual de Maringá, Av. Colombo 5790, 87020-900 Maringá-PR, Brasil, \\ ${ }^{2}$ Instituto de Química, Universidade Federal de Goiás, Campus Samambaia, Caixa Postal 131, 74001-970 \\ Goiânia-GO, Brasil, \\ ${ }^{3}$ Departamento de Ciências Farmacêuticas, Universidade Estadual de Maringá, Av. Colombo 5790, 87020-900 \\ Maringá-PR, Brasil.
}

\begin{abstract}
RESUMO: O estudo químico de Aloysia gratissima (Gillies \& Hook.) Tronc. e Aloysia virgata (Ruiz \& Pav.) Pers., Verbenaceae, resultou no isolamento de quatorze substâncias. Suas estruturas foram determinadas com base nos dados de IV, RMN de ${ }^{1} \mathrm{H}$ e de ${ }^{13} \mathrm{C}$ uni e bidimensionais e comparação com dados da literatura. A atividade antibacteriana do extrato bruto das folhas e caules e das frações de $A$. virgata, bem como as substâncias hoffmaniacetona e seu monoacetato, verbascosídeo e arenariosídeo, foram avaliadas através do método de bioautografia e a atividade antiedematogênica foi avaliada pelo modelo de edema de orelha induzido para os extratos brutos e frações metanólicas de $A$. gratissima e $A$. virgata..
\end{abstract}

Unitermos: ent-Caurano, feniletanóides, atividade antibacteriana, atividade antiedematogênica.

\begin{abstract}
Chemical composition and evaluation of antibacterial and antiedematogenic Activities of Aloysia gratissima (Gillies \& Hook.) Tronc. e Aloysia virgata (Ruiz \& Pav.) Pers., Verbenaceae". Phytochemical study of Aloysia gratissima (Gillies \& Hook.) Tronc. e Aloysia virgata (Ruiz \& Pav.) Pers., Verbenaceae, afforded fourteen compounds. The structures were established using IR, 1D and 2D NMR and by comparison of its spectroscopic data to those of literature. The antibacterial activity of the crude extract from the leaves and branches and the fractions from the crude extract of $A$. virgata, besides the isolated compounds hoffmaniacetone, hoffmaniacetone monoacetate, verbascoside and arenarioside were evaluated by means of bioautography and the antiedematogenic activity was evaluated using induced ear oedema model to the crude extracts and methanol fractions for A. gratissima and A. virgata.
\end{abstract}

Keywords: ent-Kaurane, phenylethanoids, antibacterial activity, antiedematogenic activity.

\section{INTRODUÇÃO}

Verbenaceae é uma família com cerca de 100 gêneros e aproximadamente 2600 espécies de ervas, arbustos ou pequenas árvores as quais estão distribuídas principalmente em regiões tropicais e subtropicais. Espécies desta família têm despertado interesse tanto pelo potencial biológico quanto pelo uso na medicina tradicional (Barroso, 1978).

O gênero Aloysia é constituído de aproximadamente trinta espécies de arbustos com inflorescências aromáticas conhecidas principalmente por seus óleos essenciais (Hernandez et al., 2003). Estudos fitoquímicos com folhas e caule de Aloysia triphylla levou ao isolamento de iridóides, cafeoil feniletanóides glicosilados e flavonoides (Soler et al., 1986a).
Aloysia gratissima (Gillies \& Hook.) Tronc., conhecida como alfazema-do-brasil e Aloysia virgata (Ruiz \& Pav.) Pers.,Verbenaceae, conhecida popularmente como "lixeira", são arbustos com inflorescências de cheiro agradável de distribuição predominantemente tropical e subtropical. Na literatura há registro da composição do óleo essencial destas espécies (Soler et al., 1986b; Pino et al., 2000), bem como da atividade frente à Plasmodium falciparum para A. virgata (Baelmans et al., 2000) e antimicrobiana de A. gratissima (Bailac et al., 1999).

No presente trabalho é descrito o isolamento e identificação de cinco substâncias presentes em folhas e caule de $A$. virgata, e de doze substâncias isoladas das folhas de $A$. gratissima, bem como a avaliação da atividade antibacteriana de frações de $A$. virgata e de quatro 
substâncias isoladas frente às bactérias Staphylococcus aureus (ATCC 29737), Escherichia coli (ATCC 8739), Micrococcus luteus (ATCC 9341) e Bacillus subtilis (ATCC 6633) e a avaliação da atividade antiedematogênica pelo modelo de edema de orelha induzido para os extratos brutos e frações metanólicas destas espécies.

\section{MATERIAL E MÉTODOS}

Os espectros de absorção no IV, em pastilhas de $\mathrm{KBr}$, foram registrados em espectrômetro FT-IR-BOMEM, modelo MB-series na região de 400 a $4000 \mathrm{~cm}-1$. Os espectros de $\mathrm{RMN}{ }^{1} \mathrm{H}$ e ${ }^{13} \mathrm{C}$ uni e bidimensionais (COSY, HMQC, HMBC) foram obtidos em espectrômetro Varian, modelo Mercury plus BB, $300 \mathrm{MHz}\left(300 \mathrm{MHz}\right.$ para ${ }^{1} \mathrm{H}$ e 75,5 $\mathrm{MHz}$ para ${ }^{13} \mathrm{C}$ ). Para as cromatografias em coluna (CC) utilizou-se sílica gel (0,063-0,200 mm; 70-230 mesh ASTM) ou Sephadex Lipofílico LH-20. As análises por cromatografia em camada delgada analítica (CCDA) e cromatografia em camada delgada preparativa (CCDP) foram realizadas em placas de sílica gel $\mathrm{GF}_{254}$.

\section{Material vegetal}

As folhas de Aloysia gratissima (Gillies \& Hook.) Tronc., Verbenaceae, foram obtidas na Escola de Farmácia da UFG em Goiânia/Goiás e as folhas e caules de Aloysia virgata (Ruiz \& Pav.) Pers., Verbenaceae, foram coletados às margens do Rio Meia Ponte, em Goiânia/Goiás. A identificação foi realizada pelo Prof. Heleno Dias Ferreira, do Instituto de Ciências Biológicas da Universidade Federal de Goiás. As exsicatas das espécies estão depositadas no Herbário do IBC/UFG com os números de registro \#3644 e \#24363, respectivamente.

\section{Extração e isolamento dos constituintes químicos de Aloysia virgata}

As folhas secas e moídas de $A$. virgata (107 g) foram extraídas com etanol à temperatura ambiente. $\mathrm{O}$ extrato bruto das folhas $(6,3 \mathrm{~g})$ foi suspenso em metanol (15 mL), diluído com água $(20 \mathrm{~mL})$, filtrado em florisil (10 g) e em seguida extraído com clorofórmio (2x $50 \mathrm{~mL})$. Após concentração do solvente à pressão reduzida, a massa de extrato obtida foi de $0,64 \mathrm{~g}$. Esse extrato foi submetido à cromatografia em sílica gel 60 usando-se como eluentes hexano e éter etílico em ordem crescente de polaridade. Foram obtidas 96 frações de $10 \mathrm{~mL}$ cada, reunidas em subfrações. A purificação das subfrações eluídas com hexano:éter etílico $30 \%$ e $40 \%$ forneceram as substâncias $\mathbf{1}(41,3 \mathrm{mg})$ e $\mathbf{2}(10 \mathrm{mg})$, respectivamente. O caule seco e moído (100 g) foi extraído com metanol a frio, à temperatura ambiente. $\mathrm{O}$ extrato bruto do caule $(7 \mathrm{~g})$ foi dissolvido em metanol:água (1:1) e particionado em hexano, clorofórmio e acetato de etila. A filtração da fração acetato de etila em Sephadex LH-20 com água, água:metanol 25, 50, 75\% e metanol, resultou no isolamento das substâncias 3 (35 $\mathrm{mg})$ e $\mathbf{5}(2,3 \mathrm{mg})$. A filtração da fração hidrometanólica em Sephadex LH-20 com água, água:metanol 50\% e metanol, forneceu a substância 4 (170,6 mg).

\section{Extração e isolamento dos constituintes químicos de Aloysia gratissima}

As folhas secas e moídas de A. gratissima $(855$ g) foram extraídas por maceração a frio com etanol (3x). $\mathrm{O}$ extrato bruto $(84,5 \mathrm{~g})$ foi filtrado em florisil e parte do extrato resultante $(35 \mathrm{~g})$ foi fracionado em coluna cromatográfica de sílica gel usando hexano $(400 \mathrm{~mL})$, diclorometano $(600 \mathrm{~mL})$, acetato de etila $(600 \mathrm{~mL})$ e metanol $(700 \mathrm{~mL})$ como eluentes. As frações obtidas foram concentradas em evaporador rotativo. A fração hexânica ( $1 \mathrm{~g})$, fracionada em sílica gel usando hexano e hexano:acetato de etila em gradiente de polaridade como eluentes, resultou em 73 frações de $20 \mathrm{~mL}$ cada. Da fração eluída com hexano:acetato de etila $15 \%$ isolouse o 6 (28,6 mg). A fração diclorometânica $(0,42 \mathrm{~g})$ foi submetida à cromatografia em sílica gel eluída com hexano:clorofórmio (0-100\%) e clorofórmio:metanol (0-100\%). O fracionamento resultou em 97 frações de 20 $\mathrm{mL}$ cada. A lavagem com hexano da fração eluída com clorofórmio:metanol $15 \%$ possibilitou o isolamento do triterpeno $7(15,1 \mathrm{mg})$. A fração acetato de etila $(2 \mathrm{~g})$ foi fracionada em sílica gel eluída com hexano:acetato de etila (0-100\%) resultando em onze frações de $100 \mathrm{~mL}$ cada. O estudo da fração hexano:acetato de etila $20 \%$ resultou no isolamento da substância $\mathbf{8}(9,1 \mathrm{mg})$ após recristalização com acetona, a fração hexano:acetato de etila $30 \%$ forneceu 9 (4,9 mg) após a lavagem da mesma com uma mistura de hexano:diclorometano 9:1 (v/v), a fração eluída com hexano:acetato de etila $40 \%$ foi recristalizada com acetona resultando na obtenção da substância 10 (4,4 mg), a fração hexano:acetato de etila 50\% forneceu a substância 12 (2,1 $\mathrm{mg}$ ), a fração hexano:acetato de etila $60 \%$ possibilitou o isolamento da substância $\mathbf{1 3}(5,7 \mathrm{mg})$ após lavagem com uma mistura de hexano:clorofórmio 8:2 (v/v), a fração hexano:acetato de etila $80 \%$ resultou na obtenção das substâncias 1 (6,4 mg) e $\mathbf{1 1}(9,0 \mathrm{mg})$ e o estudo da fração acetato de etila $100 \%$ resultou no isolamento da substância 14 (5,9 mg), após recristalização em acetona. Parte da fração metanólica $(530 \mathrm{mg})$ foi submetida a filtração em Sephadex LH-20 usando água, água/metanol (25, 50 e $75 \%$ ) e metanol como eluentes, obtendo-se as substâncias 3 (31,3 mg) e 4 (16,0 mg).

\section{Atividade antibacteriana}

As culturas puras das bactérias $S$. aureus (ATCC 29737), E. coli (ATCC 8739), M. luteus (ATCC 9341) e B. subtilis (ATCC 6633) foram adquiridas no Instituto de Patologia Tropical (IPTSP/UFG) e Instituto Oswaldo Cruz (Rio de Janeiro). A avaliação da atividade biológica dos 
extratos e das substâncias isoladas foi feita pelo método da bioautografia utilizando-se como padrão bactericida o cloranfenicol $(0,5 \mu \mathrm{g})$ (Betina, 1973). As amostras foram aplicadas em concentrações de $3 \mathrm{mg}$ e eluídas com mistura de solvente previamente selecionando. As cromatoplacas foram reveladas com TBTB (Thiazolyl Blue Tetrazolium Bromide). A quantificação da Atividade Inibitória Mínima (CIM) foi realizada utilizando-se o método da microdiluição em placas de 96 poços (Eloff, 1998) com concentrações variando de 1000-15,6 $\mu \mathrm{g} / \mathrm{mL}$. $\mathrm{O}$ experimento foi feito em duplicata, utilizando-se como controle positivo o cloranfenicol $(0,5 \mu \mathrm{g})$ e como corante o TBTB.

\section{Atividade antiedematogênica}

Para realização do teste antiinflamatório de Edema de Orelha Induzido foram utilizados grupos de camundongos Swiss $(n=6)$ machos, adultos, pesando entre 25-35 g. Os extratos e frações das espécies vegetais $A$. virgata e $A$. gratissima foram aplicados topicamente nas orelhas $(\mathrm{n}=6)$ nas concentrações de 5,0, 2,5 e 1,25 mg/ orelha, imediatamente após a aplicação do óleo de cróton $(200 \mu \mathrm{g})$. A indometacina (Indo), aplicada por via tópica (1 mg), foi utilizada como antiinflamatório de referência (controle positivo) e a acetona 70\% utilizada como veículo. Após 6 h, os animais foram sacrificados e, de cada orelha, foram retirados discos de $7 \mathrm{~mm}$ de diâmetro para posterior pesagem em balança analítica. $\mathrm{O}$ edema foi medido pela subtração do peso da orelha que recebeu apenas o veículo daquele que recebeu o agente irritante (Van Arman, 1974). Os experimentos foram aprovados pelo Comitê de Ética de Experimentação Animal da Universidade Estadual de Maringá (PRO 021/2009 CEAE).

\section{RESULTADOS E DISCUSSÃO}

O estudo fitoquímico das folhas e caule de $A$. virgata levou ao isolamento dos cauranos hoffmaniacetona (1) e seu acetato (2), dos feniletanóides verbascosídeo (3) e arenariosídeo (4), e do flavonol luteolina (5), enquanto que o estudo fitoquímico das folhas de A. gratissima resultou no isolamento do $\alpha$-bisabolol (6), dos triterpenos $\alpha$-amirina, ácido betulínico, ácido oleanólico e ácido ursólico (7-10), dos flavonoides genkwanina; 5-hidroxi-7,4' dimetoxiapigenina; 5-hidróxi-7,3',4'-trimetoxiluteolina e rutina (11-14), além do caurano (1) e dos feniletanóides (3) e (4). A identificação das substâncias isoladas foi feita com base nos dados espectroscópicos de IV e de RMN de ${ }^{1} \mathrm{H}$ e de ${ }^{13} \mathrm{C}$ uni e bidimensionais, e por comparação com dados da literatura.

As substâncias $\mathbf{1}$ e $\mathbf{2}$ foram isoladas como sólidos brancos com ponto de fusão $156-158{ }^{\circ} \mathrm{C}$ e $122-124{ }^{\circ} \mathrm{C}$, respectivamente. Os espectros de $\mathrm{RMN}$ de ${ }^{13} \mathrm{C}$ e de ${ }^{1} \mathrm{H}$ para a substância 1 apresentaram sinais em $\delta_{\mathrm{C}}: 217,9$ (C-3), 84,4 (C-16), 65,5 (C-17), 55,7 (C-9), 47,9 (C-14), 44,5 (C-15),
43,9 (C-13), 43,4 (C-8), 14,6 (C-20) e $\delta_{\mathrm{H}}: 3,78 / 3,64(d$, $J=11,1 \mathrm{~Hz}, \mathrm{H}-17 \mathrm{a}$ e b), 2,50 ( $d d d, J=15,9,10,8$ e $7,2 \mathrm{~Hz}$, $\mathrm{H}-2 \alpha$ ), 2,39 ( $d d d, J=15,9,7,2$ e $3,9 \mathrm{~Hz}, \mathrm{H}-2 \beta), 2,11$ ( $d d d$, $J=11,1,2,4$ e $2,1 \mathrm{~Hz}, \mathrm{H}-14 \alpha), 2,07$ (dd, $J=15,0$ e 2,1 Hz, $\mathrm{H}-15 \alpha), 0,99$ (s, H-20) característicos de diterpeno do tipo ent-3-oxo-16,17-diidroxicaurano. Os dados acima e as correlações nos espectros bidimensionais indicaram que a substância isolada de $A$. virgata e A. gratissima é estruturalmente muito próxima da calliterpenona, da hoffmaniacetona e da abeokutona (Jaensch et al., 1990), estereoisômeros que apresentam o mesmo perfil nos espectros de RMN, mas que possuem diferenças significativas nos deslocamentos de alguns sinais. A abeokutona apresenta alguns deslocamentos químicos de ${ }^{13} \mathrm{C}$ diferentes da substância isolada, principalmente em $\delta_{\mathrm{C}}$ 36,8 (C-14); 52,6 (C-15); 81,7 (C-16) e 17,7 (C-20). Para a calliterpenona, observam-se algumas diferenças entre os deslocamentos químicos de hidrogênio, que foram mais significativas em $\delta_{\mathrm{H}} 1,00-2,47(\mathrm{H}-14) ; 1,57-2,15(\mathrm{H}-15) \mathrm{e}$ 4,01-4,05 (H-17). Os dados espectroscópicos da substância 1 foram comparados aos apresentados na literatura para as três substâncias mencionadas acima. Com base nesta comparação, a substância $\mathbf{1}$ foi caracterizada como a hoffmaniacetona. A estrutura da substância $\mathbf{2}$, isolada de $A$. virgata, foi deduzida por comparação com 1 e com dados da literatura (Jaensch et al., 1990).

$\mathrm{O}$ espectro de $\mathrm{RMN}$ de ${ }^{1} \mathrm{H}$ da substância 3 apresentou sinais em $\delta_{\mathrm{H}} 7,22(d, J=2,0 \mathrm{~Hz}, \mathrm{H}-2), 6,98(d$, $J=8,1 \mathrm{~Hz}, \mathrm{H}-5), 7,16$ ( $d d, J=8,1$ e 2,0 Hz, H-6), 7,72 (d, $J=15,9 \mathrm{~Hz}, \mathrm{H}-7)$ e $6,42(d, J=15,9 \mathrm{~Hz}, \mathrm{H}-8)$ característicos de uma unidade cafeoil. Os sinais em $\delta_{\mathrm{H}} 6,87(d, J=2,0 \mathrm{~Hz}$, H-2'), 6,88 ( $d, J=8,1 \mathrm{~Hz}, \mathrm{H}-5$ ') $, 6,78$ ( $d d, J=8,1$ e 2,0 Hz, H-6'); 2,85 (m, H-7') e 3,90/4,11 (m, H-8') evidenciaram a unidade 3',4'-diidroxifeniletil. As unidades glicosídicas foram evidenciadas pelos sinais em $\delta \mathrm{H} 5,12(d, J=1,8$ $\mathrm{Hz})$ e 4,52 (d, $J=8,1 \mathrm{~Hz})$ atribuídos aos hidrogênios anoméricos da ramnose e glicose, e confirmadas pelos sinais de carbonos metílico em $\delta_{\mathrm{C}} 19,9$ e metilênico em $\delta_{\mathrm{C}}$ 63,0 , no espectro de $\mathrm{RMN}$ de ${ }^{13} \mathrm{C}$. Os sinais de carbonila em $\delta_{\mathrm{C}} 171,1$ (C-9) e de dois carbonos anoméricos em $\delta_{\mathrm{C}} 104,4$ (ramnose) e 104,9 (glicose), também foram observados. O posicionamento da unidade cafeoil no carbono C-4 da glicose foi evidenciada pelo alto valor de deslocamento químico do hidrogênio H-4 em $\delta_{\mathrm{H}} 4,98$. A posição da unidade de ramnose no carbono C-3 da glicose foi determinada pela irradiação do hidrogênio anomérico em $\delta_{\mathrm{H}} 5,12$, que provocou um aumento na intensidade do sinal em 3,87 (C-3 da glicose). A substância isolada 3 foi identificada como o verbascosídeo com base nos dados acima e por comparação com a literatura (Andary et al., 1982). A substância 4 apresentou sinais nos espectros de $\mathrm{RMN}$ de ${ }^{1} \mathrm{H}$ e de ${ }^{13} \mathrm{C}$ idênticos aos de $\mathbf{3}$, diferindo na presença de sinais adicionais de hidrogênio anomérico em dH 4,30 ( $d, J=7,8 \mathrm{~Hz})$, e de carbonos anomérico em $\mathrm{dC} 106,1$, oximetilênico em dC 67,9 e oximetínicos em $\mathrm{dC} 72,0,75,3$, e 78,3, correspondendo a uma unidade de 
xilose. O posicionamento da xilose foi evidenciado pelo alto valor de deslocamento químico apresentado pelo C-6 da glicose em $\delta_{\mathrm{C}} 70,7$ e confirmado pela correlação observada no espectro de HMBC entre o sinal deste carbono e do hidrogênio em $\delta_{\mathrm{H}} 4,30$ (H-1, xilose). A substância 4 foi identificada como o arenariosideo, através dos dados espectrais e comparação com dados da literatura (Andary \& Privat, 1985). Para as substâncias 3 e 4, a literatura relata as atividades antioxidante, antiinflamatória e antibacteriana (Didry et al., 1999; Sahpaz et al., 2002).

Sesquiterpenos, feniletanóides e iridóides são considerados metabólitos típicos de Verbenaceae. Neste trabalho foi constatado a ausência de iridóides e a presença de ent-cauranos em $A$. gratissima e A. virgata, além de flavonoides metoxilados em $A$. gratissima. Estes resultados sugerem a transferência do gênero Aloysia da família Verbenaceae para a família Lamiaceae, uma vez que esta se assemelha à Verbenaceae. A família Lamiaceae caracteriza-se pela presença de diterpenos ent-cauranos e flavonoides metoxilados, substâncias estas isoladas destas espécies (Silva et al., 2006; Oliveira, et al., 2005).

$\mathrm{O}$ extrato bruto de folhas e caule, as frações hexânica, clorofórmica, acetato de etila e metanólica de A. virgata, e as substâncias $\mathbf{1}, \mathbf{2}, \mathbf{3}$ e $\mathbf{4}$ foram avaliados frente às bactérias $S$. aureus (ATCC 29737), E. coli (ATCC 8739), M. luteus (ATCC 9341) e B. subtilis (ATCC 6633). A bioautografia mostrou que a fração clorofórmica foi ativa contra $E$. coli e $S$. aureus. As substâncias 3 e 4 mostraramse ativos contra $S$. aureus e $M$. luteus. A quantificação da atividade antibacteriana para as substâncias 3 e $\mathbf{4}$ mostrou que a CIM para a substância 3 variou de $500-250 \mu \mathrm{g} /$ $\mathrm{mL}$ para $\mathrm{S}$. aureus e $1000-500 \mu \mathrm{g} / \mathrm{mL}$ para $M$. luteus. A substância 4 mostrou-se ativo somente para $S$. aureus, em concentração que variou de $1000-500 \mu \mathrm{g} / \mathrm{mL}$. As substâncias 1 e 2 foram inativos frente a todas as bactérias testadas.

No teste de atividade antiedematogênica, os resultados foram avaliados através de análise de variância de uma via (ANOVA) e seguida do teste de Tukey. Os dados obtidos para os extratos brutos e frações metanolólicas de A. gratissima e $A$. virgata mostraram redução significativa $(p<0,05)$ na intensidade da inflamação induzida pela aplicação do óleo de cróton na orelha de camundongo, para todos os casos na concentração de $5,0 \mathrm{mg} /$ orelha, quando comparada ao grupo controle positivo (Indometacina) (Figura 1). $\mathrm{O}$ extrato bruto de A. gratissima apresentou uma redução da inflação de $23,6 \%$ em relação ao controle, enquanto que a fração metanólica mostrou uma diminuição da inflamação de $22,7 \%$. O extrato bruto e a fração metanólica de $A$. virgata apresentaram resultados semelhantes, provocando uma redução do edema de 25,1 e $21,9 \%$, respectivamente, em comparação com o controle (Tabela 1).

A presença de substâncias onde literatura relata a atividade antiinflamatória, como triterpenos, flavonoides e feniletanóides glicosídicos, e que foram obtidas no estudo químico destas espécies, podem justificar a atividade apresentada.

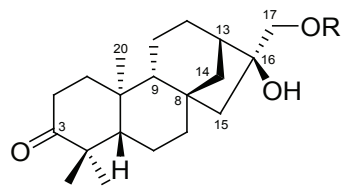

$1 \mathrm{R}=\mathrm{H}$<smiles>[R20]c1cc([R20])c2c(=O)c([R])c(-c3ccc([R6])c([R])c3)oc2c1</smiles>

$5 \mathrm{R}_{1}=\mathrm{R}_{3}=\mathrm{R}_{4}=\mathrm{H}, \mathrm{R}_{2}=\mathrm{OH}$

$11 \mathrm{R}_{1}=\mathrm{CH}_{3}, \mathrm{R}_{2}=\mathrm{R}_{3}=\mathrm{R}_{4}=\mathrm{H}$

$12 \mathrm{R}_{1}=\mathrm{R}_{3}=\mathrm{CH}_{3}, \mathrm{R}_{2}=\mathrm{R}_{4}=\mathrm{H}$

$13 \mathrm{R}_{1}=\mathrm{R}_{2}=\mathrm{R}_{3}=\mathrm{CH}_{3}, \mathrm{R}_{4}=\mathrm{H}$

$14 \mathrm{R}_{1}=\mathrm{R}_{2}=\mathrm{R}_{3}=\mathrm{H}, \mathrm{R}_{4}=\mathrm{O}=\mathrm{gli} \rightarrow \mathrm{ram}$<smiles>CC(C)=CCCC(C)(C)C1CC=C(C)CC1</smiles>

6



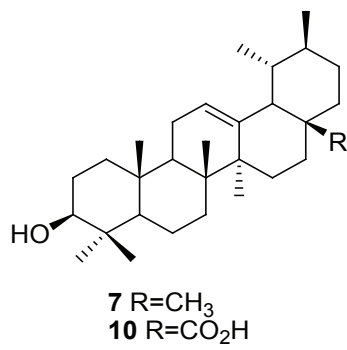<smiles>C=C(C)C1CCC2(C(=O)O)CCC3C4CCC5C(C)(C)[C@@H](O)CC[C@]5(C)C4CCC3[C@]12C</smiles>

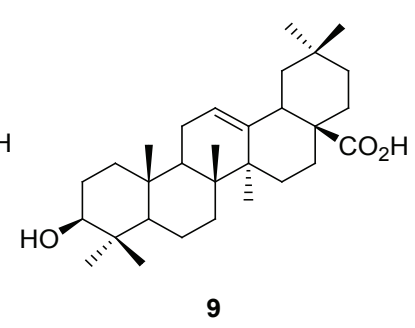




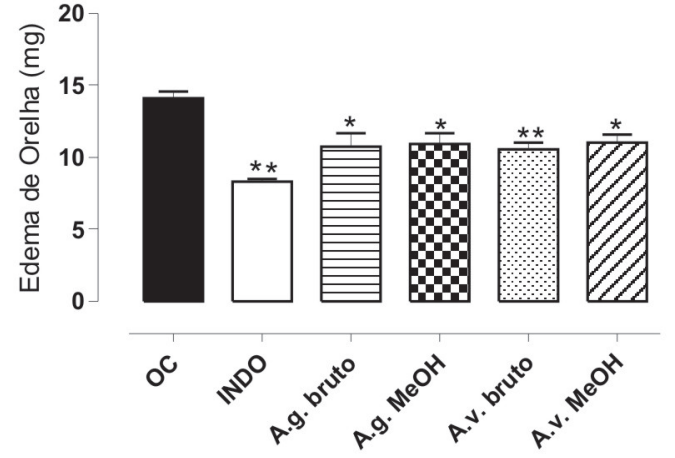

Figura 1. Efeito dos extratos brutos e frações metanólicas de $A$. gratissima e $A$. virgata sobre o edema de orelha induzido pelo óleo de cróton em camundongos.

Tabela 1. Efeito dos extratos brutos e frações metanólicas de A. gratissima e $A$. virgata na redução do edema de orelha em camundongos 6 horas após a aplicação do óleo de cróton.

\begin{tabular}{lcc}
\hline Amostra analisada & $\begin{array}{c}\text { Peso médio das } \\
\text { orelhas }(\mathrm{g})\end{array}$ & $\begin{array}{c}\text { Porcentagem de } \\
\text { redução do edema } \\
\text { de orelha provocado } \\
\text { pela aplicação de } \\
\text { óleo de cróton (\%) }\end{array}$ \\
\hline EB A. gratissima & $0,0108 \pm 8,5 \cdot 10-4$ & $23,6^{*}$ \\
FM A. gratissima & $0,0109 \pm 7,3 \cdot 10-4$ & $22,7^{*}$ \\
EB A. virgata & $0,0106 \pm 4,3 \cdot 10-4$ & $25,1^{*}$ \\
FM A. virgata & $0,0110 \pm 5,6 \cdot 10-4$ & $21,9^{*}$ \\
Indometacina & $0,00834 \pm 1,6 \cdot 10-4$ & $41,2^{*}$ \\
Óleo de cróton & $0,0141 \pm 4,1.10-4$ & \\
\hline
\end{tabular}

${ }^{*} p<0,05$; EB: Extrato bruto, FM: Fração metanólica.

\section{CONCLUSÕES}

$\mathrm{O}$ presente trabalho possibilitou o isolamento de quatorze substâncias a partir do estudo fitoquímico das folhas de Aloysia gratissima e das folhas e caules de Aloysia virgata. As substâncias foram identificadas a partir da comparação dos dados espectroscópicos obtidos com dados relatados na literatura. $\mathrm{O}$ ensaio antibacteriano realizado para as substâncias $\mathbf{1 , 2 , 3}$ e 4, mostrou que a substância 3 foi ativa frente à $S$. aureus $\mathrm{e} M$. luteus enquanto que 4 foi ativa somente frente à $S$. aureus. A avaliação da atividade antiedematogênica apresentou resultados satisfatórios, com percentual de inibição da inflamação significativa para todos os materiais testados.

\section{AGRADECIMENTOS}

Ao Prof. Ms. Heleno Dias Ferreira (Instituto de Ciências Biológicas, Universidade Federal de Goiás) pela coleta e identificação botânica das plantas, à Fundação de Apoio a Pesquisa (FUNAPE/UFG) pelo apoio financeiro $\mathrm{e}$, à CAPES, pelas bolsas concedidas.

\section{REFERÊNCIAS}

Andary C, Privat G 1985. Pheliposide et arenarioside, Deux nouveaux esters heterosidiques de l'acide cafeique isoles de Orobanche arenaria. J Nat Prod 48: 778-783.

Andary C, Wylde R, Laffite C, Privat G, Winternitz F 1982. Structures of verbascoside and orobanchoside, caffeic acid sugar esters from Orobanche rapum-genistae. Phytochemistry 21: 1123-1127.

Baelmans R, Deharo E, Bourdy G, Muñoz V, Quenevo C, Sauvain M, Ginsburg H 2000. A search for natural bioactive compounds in Bolivia through a multidisciplinary approach: Part IV. Is a new haem polymerisation inhibition test pertinent for the detection of antimalarial natural products? J Ethnopharmacol 73: 271-275.

Bailac PN, Dellacasa AD, Duschatzky CB, Firpo N, Ponzi MI 1999. Composicion del aceite esencial y actividad antimicrobiana de Aloysia gratissima de San LuisArgentina. An Assoc Quím Arg 87: 149-153.

Barroso GM 1978. Sistemática de Angiospermas do Brasil. São Paulo, EDUSP.

Betina V 1973. Bioautography in paper and thin-layer chromatography and its scope in the antibiotic field. $J$ Chromatogr 78: 41-51.

Didry N, Seidel V, Dubreuil L, Tillequin F, Bailleul F 1999. Isolation and antibacterial activity of phenylpropanoid derivatives from Ballota nigra. J Ethnopharmacol 67: 197-202.

Eloff JN 1998. A sensitive and quick microplate method to determine the minimal inhibitory concentration of plant extracts for bacteria. Planta Med 64: 711-713.

Hernandez T, Canales M, Avila JG 2003. Ethnobotany and antibacterial activity of some plants used in traditional medicine of Zapotitlán de las Salinas, Puebla (México). $J$ Ethnopharmacol 88: 181-188.

Jaensch M, Jakupovic J, Sanchez H, Dominguez XA 1990. Diterpenes from Hoffmannia strigillosa. Phytochemistry 29: 3587-3590.

Oliveira CMA, Silva CC, Ferreira HD, Lemes, GF, Schmitt E 2005. Kauranes, phenylethanoids and flavone from Aloysia virgata. Biochem Syst Ecol 33: 1191-1193.

Pino JA, Marbot R, Fuentes V 2000.Essential oil of Aloysia virgata Juss. from Cuba. J Ess Oil Res 16: 44-46.

Sahpaz S, Garbacki N, Tits M, Bailleul F 2002. Isolation and pharmacological activity of phenylpropanoid esters from Marrubium vulgare. J Ethnopharmacol 79: 389-392.

Silva CC, Vandresen F, Oliveira CMA, Kato L, Tanaka CMA, Ferreira HD 2006. Chemical composition of Aloysia gratissima (Gill. et Hook) Tronc. (Verbenaceae). Biochem Syst Ecol 34: 593-595.

Soler E, Dellacassa E, Moyna P 1986a. Iridoid glucosides as taxonomic markers in the genera Lantana, Lippia, Aloysia and Phyla. Biochem Syst Ecol 14: 307-310.

Soler E, Dellacasa E, Moyna P 1986b. Composition of Aloysia gratissima leaf essential oil. Phytochemistry 25: 13431345.

Van Arman GC 1974. Anti-inflamatory drugs. Clin Pharmacol Ther 16: 900-904. 